\title{
Determination of optimal diagnostic criteria for purulent vaginal discharge and cytological endometritis in dairy cows
}

\author{
J. Denis-Robichaud ${ }^{1}$ and J. Dubuc ${ }^{2}$ \\ Faculté de médecine vétérinaire, Université de Montréal, C.P. 5000, Saint-Hyacinthe, Québec, J2S 7C6, Canada
}

\begin{abstract}
The objectives of this observational study were to identify the optimal diagnostic criteria for purulent vaginal discharge (PVD) and cytological endometritis (ENDO) using vaginal discharge, endometrial cytology, and leukocyte esterase (LE) tests, and to quantify their effect on subsequent reproductive performance. Data generated from 1,099 untreated Holstein cows (28 herds) enrolled in a randomized clinical trial were used in this study. Cows were examined at $35( \pm 7) \mathrm{d}$ in milk for PVD using vaginal discharge scoring and for ENDO using endometrial cytology and LE testing. Optimal combinations of diagnostic criteria were determined based on the lowest Akaike information criterion (AIC) to predict pregnancy status at first service. Once identified, these criteria were used to quantify the effect of PVD and ENDO on pregnancy risk at first service and on pregnancy hazard until $200 \mathrm{~d}$ in milk (survival analysis). Predicting ability of these diagnostic criteria was determined using area under the curve (AUC) values. The prevalence of PVD and ENDO was calculated as well as the agreement between endometrial cytology and LE. The optimal diagnostic criteria (lowest AIC) identified in this study were purulent vaginal discharge or worse $(\geq 4), \geq 6 \%$ polymorphonuclear leukocytes (PMNL) by endometrial cytology, and small amounts of leukocytes or worse $(\geq 1)$ by LE testing. When using the combination of vaginal discharge and PMNL percentage as diagnostic tools $(\mathrm{n}=1,099)$, the prevalences of PVD and ENDO were 17.1 and $36.2 \%$, respectively. When using the combination of vaginal discharge and LE $(\mathrm{n}=915)$, the prevalences of PVD and ENDO were 17.1 and $48.4 \%$. The optimal strategies for predicting pregnancy status at first service were the use of $\mathrm{LE}$ only $(\mathrm{AUC}=0.578)$ and PMNL percentage only (AUC $=0.575)$. Cows affected by PVD and ENDO had 0.36 and 0.32 times the odds, respectively, of being pregnant at first service when using PMNL percentage compared
\end{abstract}

\footnotetext{
Received November 17, 2014.

Accepted March 12, 2015.

${ }^{1}$ Current address: Department of Population Medicine, University of Guelph, Guelph, Ontario, N1G 2W1, Canada.

${ }^{2}$ Corresponding author: Jocelyn.dubuc@umontreal.ca
}

with that of unaffected cows; odds ratios were 0.33 and 0.69 for PVD and ENDO, respectively, when LE was used. Kappa value (agreement) of the pairwise comparison for the PMNL percentage and LE was 0.43. Vaginal discharge, endometrial cytology, and LE can be used to diagnose PVD and ENDO, and to predict pregnancy status at first service. The use of LE could be a good alternative to endometrial cytology for onfarm testing.

Key words: dairy cow, uterine disease, endometritis, diagnosis

\section{INTRODUCTION}

Reproductive performance is a major concern in the dairy industry because of its important economic impact (LeBlanc, 2007). Reproductive tract diseases such as purulent vaginal discharge (PVD) and cytological endometritis (ENDO) are associated with detrimental effects on subsequent reproductive performance (Dubuc et al., 2010a). To implement an on-farm disease surveillance program and an immediate treatment strategy for PVD and ENDO, practical and easy-touse diagnostic tools are needed. Multiple diagnostic tools have been validated for PVD and ENDO. For example, a vaginoscope or a Metricheck device (Simcro, Hamilton, New Zealand) can be used to diagnose PVD, and both tools provide relatively similar results (McDougall et al., 2007). The diagnosis of ENDO using endometrial biopsy or cytology techniques has been validated (Kasimanickam et al., 2004; Gilbert et al., 2005; Chapwanya et al., 2010) but does not provide on-farm results. Leukocyte esterase (LE) colorimetric testing from uterine lavage or cytobrush sample was shown to provide interesting results and it was developed to provide immediate on-farm results (Cheong et al., 2012; Couto et al., 2013).

Diagnosis of PVD and ENDO requires the use of thresholds to classify the animals as diseased or not because the techniques used provide quantitative or semiquantitative results (de Boer et al., 2014). Although many studies have reported different diagnostic criteria, most of them were relatively similar (Barlund et al., 2008; Runciman et al., 2009; Dubuc et al., 
2010a). However, few studies have considered PVD and ENDO diagnosis simultaneously (de Boer et al., 2014). It is unclear at this point what the best combination of diagnostic criteria for PVD and ENDO is when taken together and whether this combination would provide better accuracy to diagnose reproductive tract disease globally. Therefore, the first objective of this study was to define PVD and ENDO diagnostic criteria using vaginal discharge and endometrial cytology in postpartum dairy cows considering both conditions simultaneously and to quantify the effect of these conditions on subsequent reproductive performance. The second objective was to validate the use of $\mathrm{LE}$ colorimetric testing as a diagnostic tool for ENDO and its effect on reproduction. The third objective was to evaluate the accuracy of these diagnostic tests for predicting the subsequent reproductive performance of dairy cows.

\section{MATERIALS AND METHODS}

A prospective cohort study was conducted from October 2011 until December 2013. A convenience sample of 28 herds was recruited in a $250-\mathrm{km}$ radius around StHyacinthe, Québec, Canada. Participating herds were required to use a computerized record system and to use an ovulation synchronization protocol to synchronize the first breeding of all cows at around 70 DIM. All herds used exclusively AI for breeding. Each cow could only be enrolled once in the study.

A total of 1,168 enrolled cows were targeted in the study. Sample size was calculated to identify a difference of $10 \%$ in pregnancy risk at first service between diseased (20\%) and nondiseased (30\%) cows with $95 \%$ confidence and $80 \%$ power (Dohoo et al., 2009) when expecting an overall disease prevalence of $25 \%$. The present study was part of a larger research project involving a randomized clinical trial of intrauterine cephapirin treatment for endometritis in postpartum dairy cows. All cows enrolled in the present study were control animals and did not receive any intrauterine treatment that could influence their reproductive performance. Cows that were not bred before 100 DIM were excluded from the project.

Farms were visited every other week by an animal health technician and a veterinarian. All participating cows were examined for PVD and ENDO at $35( \pm 7)$ DIM. First, vaginal discharge was evaluated with the Metricheck device technique (McDougall et al., 2007) and scoring $(0=$ no discharge, $1=$ clear mucus, $2=$ mucus with flecks of pus, $3=$ mucopurulent discharge, $4=$ purulent discharge or $5=$ foul-smelling discharge). A cytological sample was then collected from the uterus using a cytobrush (VWR CanLab, Mississauga, Canada) adapted for use in cattle (Kasimanickam et al., 2004; Dubuc et al., 2010a). After collection, the cytobrush was rolled on a clean glass microscope slide and plunged into an individual vial $(3 \mathrm{~mL})$ containing $1 \mathrm{~mL}$ of $0.9 \%$ saline ( $\mathrm{NaCl} 0.9 \%$ Irrigation, Baxter Corp., Mississauga, ON, Canada). Within $12 \mathrm{~h}$ of collection, endometrial cytology slides were stained with a modified Wright-Giemsa stain (Hema3, Biochemical Sciences, Swedesboro, NJ) and coverslips were applied when dry (Barlund et al., 2008). Microscopic evaluation of cytology slides was done separately at $400 \times$ magnification by 2 experienced observers (animal health technician and veterinarian; Kasimanickam et al., 2005). Percentage of PMNL was determined in 2 different regions of each slide based on a differential count of 200 cells (polymorphonuclear and endometrial cells). Slide readers were blinded to on-farm clinical findings.

Within $12 \mathrm{~h}$ of collection, LE testing was performed by shaking the individual vial for $10 \mathrm{~s}$ and putting a drop of the solution on an LE commercial test strip (Multistix 10 SG, Bayer Corporation, Elkart, IN) with a pipette. Test results for every cow were read according to the manufacturer's colorimetric chart after $2 \mathrm{~min}$, and the score was recorded as follows: $0=$ negative, 0.5 $=$ trace of leukocytes, $1=$ small amount of leukocytes, $2=$ moderate amount of leukocytes, and $3=$ large amount of leukocytes (Couto et al., 2013). Test readers were blinded to the on-farm disease clinical findings.

\section{Statistical Analyses}

All statistical analyses were performed using SAS version 9.3 (SAS Institute Inc., Cary, NC). The cow was considered as the unit of interest in this study. Individual cow data such as parity (first, second, and third or greater), season of calving (winter: January to March; spring: April to June; summer: July to September; fall: October to December), and reproductive and culling events were exported from a DSA computerized record system (DSAHR Inc., Saint-Hyacinthe, QC, Canada) into Microsoft Excel (Microsoft Corp., Redmond, WA).

Diagnostic Criteria. To determine to optimal diagnostic criteria for PVD and ENDO, a dichotomous dummy variable was created for each disease (PVD and ENDO). When the score of vaginal discharge was at or above the vaginal discharge diagnostic criterion, the variable PVD was considered positive, regardless of the cytology result. If the percentage of PMNL was at or above the PMNL diagnostic criterion, the variable ENDO was considered positive. All possible combinations of PVD and ENDO diagnostic criteria were tested in different logistic regression models predicting the probability of nonpregnancy at first service 
and adjusted for herd clustering (random effect; PROC GLIMMIX) as follows:

$$
\begin{gathered}
\mathrm{Y}_{\mathrm{ij}} \sim \operatorname{bin}\left[P\left(\mathrm{Y}_{\mathrm{ij}}\right)\right] \\
\operatorname{Logit}\left[P\left(\mathrm{Y}_{\mathrm{ij}}\right)\right]=\beta_{0 \mathrm{ij}}+\beta_{1} \mathrm{PVD}_{\mathrm{ij}}+\beta_{2} \mathrm{ENDO}_{\mathrm{ij}}+\mu_{\mathrm{j}},
\end{gathered}
$$

where $P\left(\mathrm{Y}_{\mathrm{ij}}\right)$ is the probability of nonpregnancy at first service of cow $i$ in herd $j$ and is function of the diagnosed uterine status through the logit function; $\beta_{0 \mathrm{ij}}$ is the intercept; $\beta_{1}$ is the regression coefficient for PVD status (determined using a given vaginal discharge threshold); $\beta_{2}$ is the regression coefficient for ENDO status (determined using a given PMNL or LE threshold); and $\mu_{\mathrm{j}}$ is the random herd effect.

Models with different combinations of vaginal discharge (2 to 4 ) and PMNL percentage (1 to $20 \%$ ) diagnostic criteria were computed and the one with the lowest Akaike information criterion (AIC) was selected (Burnham and Anderson, 1998). The value of AIC can be used to compare non-nested models, and better models provide lower AIC values than poorer models (Burnham and Anderson, 1998; Dohoo et al., 2009). These models were built to identify the combination of diagnostic criteria that had the best ability to predict nonpregnancy at first service. The same procedure was performed for the combination of vaginal discharge (2 to 4 ) and LE (0.5 to 4 ) results. These models yielded 2 pairs of optimal diagnostic criteria. These 2 pairs were retained and used in all subsequent analyses. Based on these optimal diagnostic criteria, dichotomous variables were created for PVD and ENDO.

Endometritis Prevalence. The prevalence of PVD and ENDO was calculated (PROC FREQ). Herd prevalence of PVD and ENDO was also calculated (PROC FREQ).

Predicting Ability. The ability of optimal thresholds for predicting non-pregnancy at first service was computed. Two approaches were used: (1) the optimal combination of VD and PMNL results; (2) the optimal combination of VD and LE results. Evaluated test characteristics included the area under the curve (AUC; obtained using logistic regression models; PROC LOGISTIC), the sensitivity (Se), specificity (Sp), and positive and negative predictive values (PPV and NPV, respectively; obtained using $2 \times 2$ tables and asymptotic confidence intervals; PROC FREQ).

To compare the results from LE and cytology, the kappa statistic (agreement beyond chances) was calculated (PROC FREQ) between the 2 tests at their optimal thresholds. Interobserver agreement was calculated in a random subsample of 100 results for VD, LE, and cytology, and intraobserver agreement was calculated for 100 endometrial cytology slides (Dohoo et al., 2009).
Effect of $P \boldsymbol{P} \boldsymbol{D}$ and $\boldsymbol{E N D O}$. The effect of PVD and ENDO on subsequent reproductive performance was assessed based on pregnancy risk at first service and on pregnancy hazard during the first 200 DIM (survival analysis). For pregnancy risk at first service, a logistic regression model accounting for herd clustering as a random effect (PROC GLIMMIX) was used. For pregnancy hazard, a Cox proportional hazard model accounting for herd clustering by shared frailty (PROC PHREG) was used. Parity and season of calving were offered to all models as confounders, and were kept as fixed effects if their effect on the model estimates was greater than 10\% (Maldonado and Greenland, 1993).

\section{RESULTS}

A total of 1,099 Holstein cows from 28 herds were enrolled in the present study and examined for vaginal discharge and PMNL percentage; LE testing was performed on only 914 cows because of a delay in obtaining material at the start of the study. Herd size ranged from 40 to 600 cows, and most herds $(\mathrm{n}=22)$ were housed in tiestall facilities (6 herds were housed in freestall housing).

\section{Diagnostic Criteria}

The combinations of diagnostic criteria that had the optimal ability to predict nonpregnancy at first service (lowest AIC) were vaginal discharge $\geq 4$ (purulent discharge or worse) and PMNL percentage $\geq 6 \%$ (Table 1 ), and vaginal discharge $\geq 4$ (purulent discharge or worse) and LE $\geq 1$ (small amounts of leukocytes; Table 2).

\section{Endometritis Prevalence}

The distribution of PVD and ENDO (using PMNL percentage or LE) cases is presented in Table 3. Prevalence of PVD was $17.1 \%(\mathrm{n}=188)$ in this study. Prevalence of ENDO was 36.2\% ( $\mathrm{n}=398)$ when using PMNL percentage and $48.4 \%(\mathrm{n}=443)$ when using LE. Herd prevalence ranged from 4 to $29 \%$ for PVD, from 13 to $64 \%$ for ENDO when diagnosed by PMNL percentage, and from 26 to $89 \%$ when diagnosed by LE.

\section{Predicting Ability}

Table 4 presents the diagnostic test characteristics (AUC, Se, Sp, PPV, NPV) for predicting pregnancy status at first service using various test strategies (alone or in combination). The highest AUC results were observed for the use of LE alone (0.578) and PMNL percentage alone (0.575) 
Table 1. Akaike information criterion (AIC) values and prevalence of purulent vaginal discharge (PVD) and cytological endometritis (ENDO) for models predicting pregnancy status at first service for various diagnostic criteria combinations at $35( \pm 7)$ DIM in 1,099 Holstein dairy cows ${ }^{1}$

\begin{tabular}{|c|c|c|c|c|c|}
\hline \multicolumn{2}{|c|}{ Diagnostic criteria } & \multirow[b]{2}{*}{ AIC value } & \multicolumn{3}{|c|}{ First-service pregnancy risk (\%) } \\
\hline $\begin{array}{l}\text { Vaginal } \\
\text { discharge }^{2}\end{array}$ & $\begin{array}{l}\text { Cytology } \\
\text { (\% PMNL) }\end{array}$ & & PVD & ENDO & Unaffected \\
\hline \multirow[t]{8}{*}{$\geq 2$} & $\geq 1$ & $1,271.5$ & 21.7 & 30.1 & 34.5 \\
\hline & $\geq 2$ & $1,271.4$ & 21.7 & 29.4 & 33.7 \\
\hline & $\geq 4$ & $1,271.5$ & 21.7 & 28.6 & 32.7 \\
\hline & $\geq 6$ & $1,266.1$ & 21.7 & 28.4 & 32.7 \\
\hline & $\geq 8$ & $1,270.5$ & 21.7 & 26.8 & 33.0 \\
\hline & $\geq 10$ & $1,271.8$ & 21.7 & 25.4 & 32.8 \\
\hline & $\geq 14$ & $1,268.7$ & 21.7 & 16.5 & 32.3 \\
\hline & $\geq 18$ & $1,262.2$ & 21.7 & 12.6 & 33.0 \\
\hline \multirow{8}{*}{$\geq 3$} & $\geq 1$ & $1,262.8$ & 22.5 & 26.7 & 34.5 \\
\hline & $\geq 2$ & $1,263.3$ & 22.5 & 25.4 & 33.7 \\
\hline & $\geq 4$ & $1,274.7$ & 22.5 & 22.1 & 32.4 \\
\hline & $\geq 6$ & $1,272.0$ & 22.5 & 17.4 & 33.2 \\
\hline & $\geq 8$ & $1,261.8$ & 22.5 & 17.7 & 32.1 \\
\hline & $\geq 10$ & $1,267.0$ & 22.5 & 14.3 & 31.7 \\
\hline & $\geq 14$ & $1,267.1$ & 22.5 & 9.9 & 31.5 \\
\hline & $\geq 18$ & $1,259.3$ & 22.5 & 9.0 & 31.0 \\
\hline \multirow[t]{8}{*}{$\geq 4$} & $\geq 1$ & $1,264.8$ & 15.0 & 27.2 & 34.0 \\
\hline & $\geq 2$ & $1,261.7$ & 15.0 & 25.8 & 34.0 \\
\hline & $\geq 4$ & $1,246.4$ & 15.0 & 21.2 & 34.0 \\
\hline & $\geq 6$ & $1,240.0$ & 15.0 & 16.3 & 35.0 \\
\hline & $\overline{\geq 8}$ & $1,242.9$ & 15.0 & 16.0 & 33.8 \\
\hline & $\geq 10$ & $1,245.0$ & 15.0 & 16.3 & 33.2 \\
\hline & $\geq 14$ & $1,256.3$ & 15.0 & 13.5 & 33.1 \\
\hline & $\geq 18$ & $1,260.1$ & 15.0 & 9.0 & 32.8 \\
\hline
\end{tabular}

${ }^{1}$ All models were adjusted for herd clustering (random effect). Optimal pair of diagnostic criteria (model with lowest AIC) is presented in bold.

${ }^{2}$ Metricheck (Simcro, Hamilton, New Zealand) vaginal scoring $(0=$ no discharge, $1=$ clear mucus, $2=$ mucus with flecks of pus, $3=$ mucopurulent discharge, $4=$ purulent discharge and $5=$ foul smelling discharge).

Table 2. Akaike information criterion (AIC) values and prevalence of purulent vaginal discharge (PVD) and cytological endometritis (ENDO) for models predicting pregnancy status at first service for various diagnostic criteria combinations at $35( \pm 7)$ DIM in 915 Holstein dairy cows ${ }^{1}$

\begin{tabular}{|c|c|c|c|c|c|}
\hline \multicolumn{2}{|c|}{ Diagnostic criteria } & \multirow[b]{2}{*}{ AIC value } & \multicolumn{3}{|c|}{ First-service pregnancy risk (\%) } \\
\hline $\begin{array}{l}\text { Vaginal } \\
\text { discharge }^{2}\end{array}$ & $\begin{array}{l}\text { Leukocyte } \\
\text { esterase }^{3}\end{array}$ & & PVD & ENDO & Unaffected \\
\hline \multirow[t]{4}{*}{$\geq 2$} & $\geq 0.5$ & $1,267.8$ & 21.6 & 27.8 & 35.6 \\
\hline & $>1$ & 1.264 .5 & 21.6 & 23.7 & 34.2 \\
\hline & $\geq 2$ & $1,260.7$ & 21.6 & 18.4 & 33.4 \\
\hline & $\geq 3$ & $1,265.3$ & 21.6 & 18.2 & 31.2 \\
\hline \multirow[t]{4}{*}{$\geq 3$} & $\overline{\geq} 0.5$ & $1,267.7$ & 22.5 & 25.6 & 33.7 \\
\hline & $\overline{\geq} 1$ & $1,271.9$ & 22.5 & 21.8 & 32.1 \\
\hline & $\geq 2$ & $1,268.1$ & 22.5 & 17.4 & 31.3 \\
\hline & $\geq 3$ & $1,264.3$ & 22.5 & 15.9 & 29.1 \\
\hline \multirow[t]{4}{*}{$\geq 4$} & $\geq 0.5$ & $1,271.6$ & 16.3 & 26.3 & 35.1 \\
\hline & $>_{1}$ & $1,259.2$ & 16.3 & 23.4 & 32.4 \\
\hline & $\geq 2$ & $1,261.2$ & 16.3 & 18.3 & 32.6 \\
\hline & $>3$ & $1,270.3$ & 16.3 & 15.9 & 30.4 \\
\hline
\end{tabular}

${ }^{1}$ All models were adjusted for herd clustering (random effect). Optimal pair of diagnostic criteria (model with lowest AIC) is presented in bold.

${ }^{2}$ Metricheck (Simcro, Hamilton, New Zealand) vaginal scoring $(0=$ no discharge, $1=$ clear mucus, $2=$ mucus with flecks of pus, $3=$ mucopurulent discharge, $4=$ purulent discharge and $5=$ foul smelling discharge).

${ }^{3}$ Leukocyte esterase scoring $(0=$ negative, $0.5=$ trace of leukocytes, $1=$ small amount of leukocytes, $2=$ moderate amount of leukocytes, and $3=$ large amount of leukocytes). 
Table 3. Prevalence of purulent vaginal discharge (purulent discharge or worse; $\geq 4$ ) and of cytological endometritis (PMNL $\geq 6 \%$ or small amount of leukocytes or worse; $\geq 1$ ) in Holstein dairy cows at $35( \pm 7)$ DIM

\begin{tabular}{lcr}
\hline & \multicolumn{2}{c}{ Purulent vaginal discharge } \\
\cline { 2 - 3 } Item & $\begin{array}{c}\text { Negative } \\
\text { No. }(\%)\end{array}$ & \multicolumn{1}{c}{$\begin{array}{c}\text { Positive } \\
\text { No. }(\%)\end{array}$} \\
\hline Cytological endometritis PMNL \% $(\mathrm{n}=1,099)$ & $622(56.6)$ & $79(7.2)$ \\
$\quad$ Negative & $289(26.3)$ & $109(9.9)$ \\
Positive & & $58(6.3)$ \\
Leukocyte esterase $(\mathrm{n}=915)$ & $414(45.2)$ & $99(10.9)$ \\
$\quad$ Negative & $344(37.6)$ & \\
\hline
\end{tabular}

Kappa value (agreement beyond chances) of pairwise comparison for PMNL percentage and LE was 0.43 (95\% CI: 0.37-0.48). Interobserver agreement (kappa) was 0.786 (95\% CI: 0.635-0.936) for vaginal discharge, 0.780 (95\% CI: $0.503-0.956$ ) for PMNL percentage, and 0.920 (95\% CI: 0.844-0.997) for LE. Intraobserver agreement (kappa) for each observer $(\mathrm{n}=2)$ were 0.761 (95\% CI: $0.555-0.827)$ and 0.744 (95\% CI: 0.507-0.926) for PMNL percentage.

\section{Effect of PVD and ENDO}

When using the test combination of vaginal discharge and PMNL percentage, PVD (odds ratio: 0.36; 95\% CI: $0.21-0.54 ; P<0.01$ ) and ENDO (odds ratio: $0.32 ; 95 \%$ CI: $0.19-0.51 ; P<0.01)$ were associated with first-service pregnancy risk. In survival analysis, PVD (hazard ratio: $0.79 ; 95 \%$ CI: $0.63-0.98 ; P=0.03)$ and ENDO (hazard ratio: $0.72 ; 95 \%$ CI: $0.59-0.87 ; P<0.01$ ) were associated with pregnancy hazard until 200 DIM.

When using the test combination of vaginal discharge and LE, PVD (odds ratio: 0.33; 95\% CI: 0.19-0.47; $P<$ 0.01 ) and ENDO (odds ratio: 0.69; 95\% CI: 0.39-0.98;
$P=0.04)$ were associated with first-service pregnancy risk. In survival analysis, PVD (hazard ratio: $0.75 ; 95 \%$ CI: $0.58-0.96 ; P=0.03$ ) and ENDO (hazard ratio: $0.80 ; 95 \%$ CI: $0.65-0.98 ; P=0.04)$ were associated with pregnancy hazard until 200 DIM.

To complete this information, Figure 1 presents the effect of reproductive tract health status (PVD, ENDO, or unaffected) on first-service pregnancy risk (least squares means) stratified based on using PMNL percentage or LE for ENDO diagnosis.

\section{DISCUSSION}

\section{Diagnostic Criteria}

Purulent vaginal discharge and ENDO have usually been considered separately to determine the optimal diagnostic criteria associated with an altered subsequent reproductive performance (de Boer et al., 2014). In most cases, studies have investigated only one form of the disease (PVD or ENDO; LeBlanc et al., 2002; Kasimanickam et al., 2004; McDougall et al., 2007). The present study is one of the first to identify the

Table 4. Test characteristics [area under the curve (AUC), sensitivity (Se), specificity (Sp), positive and negative predictive values (PPV, NPV)] of vaginal discharge (VD), PMNL, and leukocyte esterase (LE) used individually or in combination in Holstein cows at 35 ( \pm 7 ) DIM to predict nonpregnancy status at first service

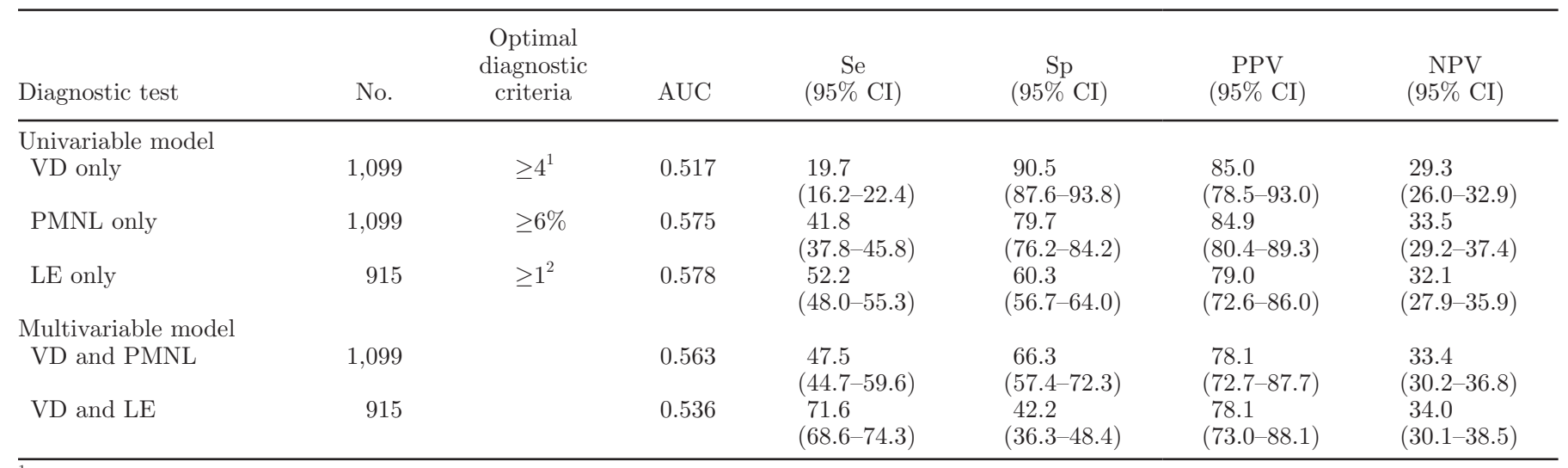

${ }^{1}$ Purulent vaginal discharge or worse.

${ }^{2}$ Small amounts of leukocytes or worse. 
diagnostic criteria that optimize the ability to predict nonpregnancy at first service when considering both conditions simultaneously; such an approach allows for accounting for their potential synergistic effect on reproductive performance (Dubuc et al., 2010a). The use of information theoretic criteria such as the AIC allows the comparison of non-nested models and the selection of the optimal fitted model by selecting the minimal value of AIC (Burnham and Anderson, 1998). When using such an approach for establishing reproductive tract disease diagnostic criteria, the present study found a diagnostic criterion of greater severity (VD $\geq 4$; purulent discharge or worse) for PVD than previous studies, which generally reported a diagnostic criterion of mucopurulent or worse vaginal discharge (VD $\geq 3$; LeBlanc et al., 2002; Williams et al., 2005; Dubuc et al., 2010a). For ENDO, diagnostic criteria reported by previous studies for endometrial cytology generally ranged between $\geq 5$ and $\geq 8 \%$ PMNL (Barlund et al., 2008; Dubuc et al., 2010a; Deguillaume et al., 2012). Our results were similar to these studies. If using LE for diagnosing ENDO, studies reported that the optimal diagnostic criteria would be moderate or worse ( $\mathrm{LE} \geq 2$ ) amount of leukocytes (Cheong et al., 2012; Couto et al., 2013). Our results suggested a lower threshold ( $\mathrm{LE} \geq 1$; small amounts of leukocytes or worse) for diagnosing ENDO when accounting for vaginal discharge.

Based on the fact that our diagnostic criteria were relatively similar to those previously reported, it suggests that previous studies have provided accurate results, even though they did not consider both PVD and ENDO diagnoses simultaneously. On the other hand, it also suggests that using information theoretic criteria such as the AIC to identify the optimal pair of diagnostic criteria is an interesting and relevant procedure. In fact, such an approach could be useful to identify optimal thresholds in future studies when comparing non-nested models. This approach is also consistent with a suggestion made by de Boer et al. (2014) relative to using objective methods to define endometritis. The use of AIC could apply to endometritis studies but also to other topics using 2 or more diagnostic tools.

\section{Endometritis Prevalence}

The overall prevalence of PVD in our study was $17.1 \%$, which is similar to values of 10 to $19 \%$ as reported by other studies (LeBlanc et al., 2002; Barlund et al., 2008; Dubuc et al., 2010a). Herd prevalence of PVD in the current study was variable (4 to 29\%); such variation would likely be caused by the presence of some risk factors for PVD in these herds. Some of these risk factors could include retained placenta, dystocia, and twinning (Dubuc et al., 2010b). The present study was not designed to determine what an appropriate PVD herd-level prevalence would be. However, it appears that prevalence between 10 and $17 \%$ is the average found in many studies around 30 to 40 DIM (LeBlanc et al., 2002; Barlund et al., 2008; Dubuc et al., 2010a).

The overall prevalence of ENDO in our study was $36.2 \%$ when using endometrial cytology. Such prevalence is higher than what has been reported in some previous studies (Barlund et al., 2008; Dubuc et al., 2010a; Cheong et al., 2011) and lower than others (Gilbert et al., 1998, 2005). It is unclear why the ENDO

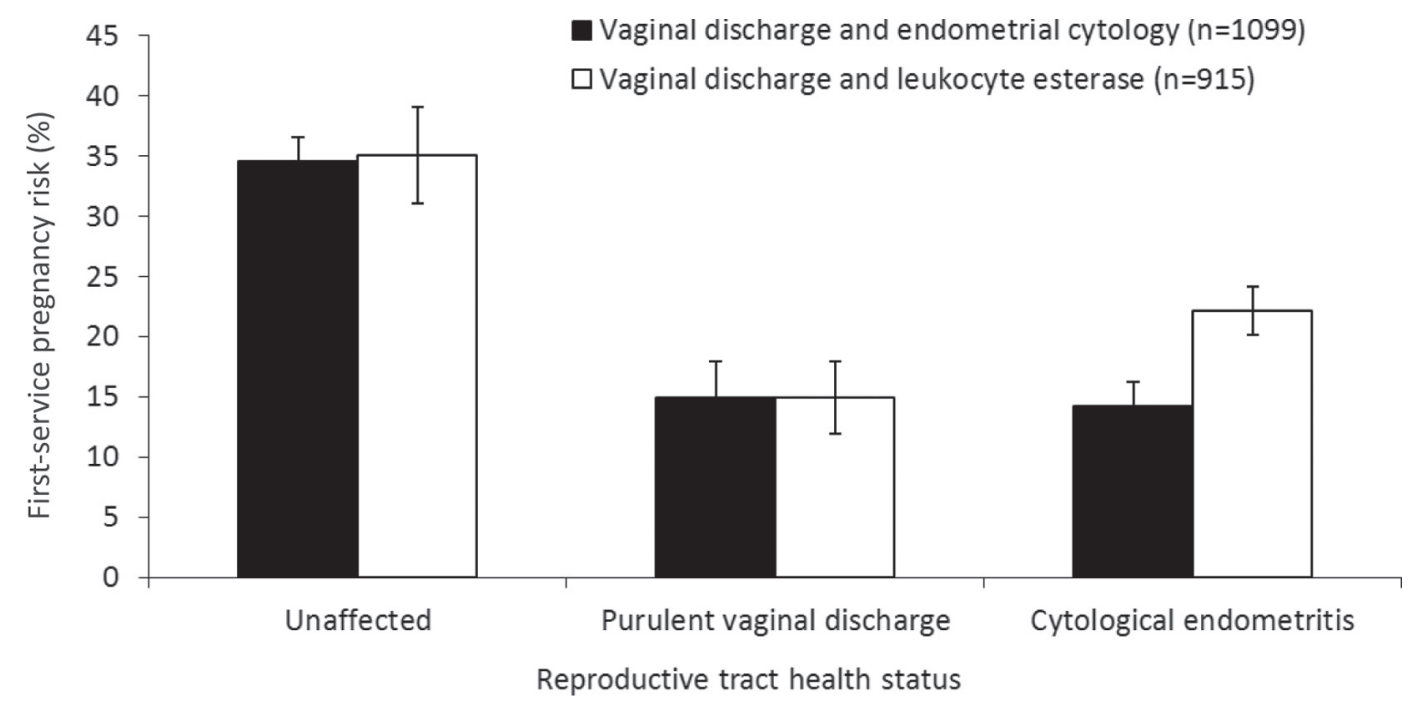

Figure 1. Effect of reproductive tract health status (purulent vaginal discharge, cytological endometritis, or unaffected) on first-service pregnancy risk in Holstein cows at $35( \pm 7)$ DIM stratified by the diagnostic methods used (vaginal discharge plus cytology or vaginal discharge plus leukocyte esterase). 
prevalence is highly variable between studies. Interestingly, it appears that studies conducted on a relatively small number of herds ( $<10$ herds) reported quite high or low prevalence (Gilbert et al., 2005; Barlund et al., 2008; Dubuc et al., 2010a). However, studies conducted on a greater number of herds, such as 38 herds (Cheong et al., 2011) and 28 herds (the present study), reported a prevalence between 25 and $36 \%$. Perhaps, a selection bias occurred during the recruitment process; herds selected for studies with a small number would generally be those selected by convenience that would have either excellent cow management (e.g., for record keeping) or poor management (ideal to find a great number of cows with endometritis). When enrolling a greater number of herds, it would be more difficult to recruit only good or poor management herds. In the end, because most of these studies (including the present one) were not designed for herd-level prevalence studies, it should be kept in mind that the reported prevalence data are only useful to provide an idea of the types of herds enrolled.

The overall prevalence of ENDO in our study was $48.4 \%$ when using LE. The study of Cheong et al. (2012) reported a prevalence of $56 \%$ when a threshold of moderate or worse $(\mathrm{LE} \geq 2)$ leukocyte numbers was used. In our study, the optimal LE criterion was LE $\geq 1$ (small amounts of leukocytes). We expected that the prevalence in our study would be higher than that in Cheong et al. (2012) because we used a lower threshold, but this was not the case. Interestingly, both studies reported that their diagnostic criteria were associated with subsequent reproductive performance. At this time, we cannot explain the discrepancy. Potential reasons could be herd selection bias, comparing 2 different uterine sampling techniques (endometrial cytology vs. low-volume uterine lavage), or accounting or not for the presence of purulent vaginal discharge. Future studies should investigate this finding to propose acceptable herd-level LE prevalence.

Although it was previously assumed that endometrial inflammation was generally present in cases of PVD, only 55\% (109 cows with positive cytology out of 188 cows with positive VD) of cows were at or above the diagnostic criteria identified for ENDO using cytology. This observation was also reported by Dubuc et al. (2010a); they reported that $38 \%$ of cows with PVD were at or above the diagnostic criteria for ENDO.

\section{Predicting Ability}

In this study, the ability of PVD and CYTO to predict nonpregnancy at first service was assessed using AUC, Se, Sp, PPV, and NPV values. It is notable that the AUC values were between 0.5 and 0.6 , which is only slightly better than chance. These results may be explained by the delay between reproductive tract testing and first service. It could also be explained by the fact that multiple factors other than uterine health can affect pregnancy risk at first service (LeBlanc, 2005). However, the test that predicted nonpregnancy at first service the most accurately (highest AUC; Faraggi and Reiser, 2002) was LE followed by PMNL percentage. Our results suggest that the use of LE for diagnosis of ENDO is an interesting and practical alternative to endometrial cytology. To provide immediate on-farm results, LE testing could be performed on farms instead of at the veterinary clinic.

A substantial agreement (Dohoo et al., 2009) between PMNL percentage and LE was expected in our study results based on the kappa value of 0.60 reported in a preliminary study by Santos et al. (2006). Surprisingly, only a moderate agreement between PMNL percentage and LE (kappa $=0.43$ ) was found. This finding could suggest that endometrial cytology and LE do not provide the same information about uterine inflammation. Nonetheless, both can be useful ENDO diagnostic tools as they are both associated with detrimental effects on subsequent reproductive performance.

\section{Effect of PVD and ENDO}

As expected, cows with positive vaginal discharge, PMNL percentage, and LE test results had lower pregnancy hazard (survival analysis) than cows with negative test results. Similar effects were reported in other studies (LeBlanc et al., 2002; Dubuc et al., 2010a). The magnitude of the effect of PVD and ENDO on firstservice pregnancy risk in the present study was between -10 percentage points (when using vaginal discharge and LE results) and -20 percentage points (when using vaginal discharge and PMNL percentage). Interestingly, this magnitude was greater than expected at the start of the study, as -10 percentage points was used in the sample size calculation. The odds of being pregnant at first service for cows affected by PVD and ENDO were 0.36 and 0.32 , respectively, in the present study compared with cows unaffected by these conditions. Similar studies by LeBlanc et al. (2002; PVD) and Kasimanickam et al. (2004; ENDO) reported odds ratios of 0.69 and 0.68 , respectively. From a survival analysis point of view, cows affected by PVD and ENDO had 0.72 to 0.79 times the daily hazard of pregnancy compared with cows unaffected by these conditions. Our results were similar to the values of 0.73 and 0.59 reported by LeBlanc et al. (2002; PVD) and Kasimanickam et al. (2004; ENDO), respectively.

To improve the quality of study design and reporting, a checklist from de Boer et al. (2014) was used in the present study to provide all relevant information for 
potential future systematic reviews (see list at http:// dx.doi.org/10.3168/jds.2013-7450). A common lack of reporting for some specific points was highlighted in that paper (de Boer et al., 2014), including inter- and intraobserver test agreement; these points were addressed in this study.

\section{CONCLUSIONS}

Vaginal discharge, PMNL percentage, and LE can be used to diagnose PVD and ENDO in dairy cows. The optimal diagnostic criterion for PVD was purulent vaginal discharge $(\mathrm{VD} \geq 4)$, whereas the optimal diagnostic criterion for ENDO was $\geq 6 \%$ PMNL at endometrial cytology or small amounts of leukocytes $(\geq 1)$ using LE testing. Using these definitions, PVD and ENDO were associated with detrimental effects on subsequent reproductive performance. The use of LE provided interesting results and could be considered an on-farm alternative to endometrial cytology. These diagnostic tools could be used for the surveillance of endometritis in dairy herds, but the acceptable prevalence at the herd level deserves further investigation.

\section{ACKNOWLEDGMENTS}

The authors acknowledge Merck Animal Health (Montreal, Canada) for funding this research project. José Denis-Robichaud was financially supported by scholarships from Novalait (Longueuil, Canada), from the Fond de recherche du Québec en nature et technologie (FRQNT; Quebec, Canada), and from the Conseil canadien de recherche en sciences naturelles et génie (CRSNG; Ottawa, Canada). The authors also thank the participating farmers for their willingness to contribute to this study. The technical support provided by Jean-Philippe Pelletier, Christine Ouellet, and Catherine B. Loubier (University of Montreal, St-Hyacinthe, Canada) is greatly appreciated.

\section{REFERENCES}

Barlund, C. S., T. D. Carruthers, C. L. Waldner, and C. W. Palmer. 2008. A comparison of diagnostic techniques for postpartum endometritis in dairy cattle. Theriogenology 69:714-723.

Burnham, K. P., and D. R. Anderson. 1998. Model Selection and Multimodel Inference. 2nd ed. Springer, New York, NY.

Chapwanya, A., K. G. Meade, F. Narciandi, P. Stanley, J. F. Mee, M. L. Doherty, J. J. Callanan, and C. O'Farrelly. 2010. Endometrial biopsy: A valuable clinical and research tool in bovine reproduction. Theriogenology 73:988-994.

Cheong, S. H., D. V. Nydam, K. N. Galvao, B. M. Crosier, and R. O. Gilbert. 2011. Cow-level and herd-level risk factors for subclinical endometritis in lactating Holstein cows. J. Dairy Sci. 94:762-770.
Cheong, S. H., D. V. Nydam, K. N. Galvao, B. M. Crosier, A. Ricci, L. S. Caixeta, R. B. Sper, M. Fraga, and R. O. Gilbert. 2012. Use of reagent test strips for diagnosis of endometritis in dairy cows. Theriogenology 77:858-864.

Couto, G. B., D. H. Vaillancourt, and R. C. Lefebvre. 2013. Comparison of a leukocyte esterase test with endometrial cytology for diagnosis of subclinical endometritis in postpartum dairy cows. Theriogenology 79:103-107.

de Boer, M. W., S. J. LeBlanc, J. Dubuc, S. Meier, H. Heuwieser, S. Arlt, R. O. Gilbert, and S. McDougall. 2014. Invited review: Systematic review of diagnostic tests for reproductive-tract infection and inflammation in dairy cows. J. Dairy Sci. 97:3983-3999. http://dx.doi.org/10.3168/jds.2013-7450.

Deguillaume, L., A. Geffré, L. Desquilbet, A. Dizien, S. Thoumire, C. Vornière, F. Constant, R. Fournier, and S. Chastant-Maillard. 2012. Effect of endocervical inflammation on days to conception in dairy cows. J. Dairy Sci. 95:1776-1783.

Dohoo, I. R., S. W. Martin, and H. Stryhn. 2009. Veterinary Epidemiologic Research. 2nd ed. VER Inc., Charlottetown, PE, Canada.

Dubuc, J., T. F. Duffield, K. E. Leslie, J. S. Walton, and S. J. LeBlanc. 2010a. Definitions and diagnosis of postpartum endometritis in dairy cows. J. Dairy Sci. 93:5225-5233.

Dubuc, J., T. F. Duffield, K. E. Leslie, J. S. Walton, and S. J. LeBlanc. 2010b. Risk factors for postpartum uterine diseases in dairy cows. J. Dairy Sci. 93:5764-5771.

Faraggi, D., and B. Reiser. 2002. Estimation of the area under the ROC curve. Stat. Med. 21:3093-3106.

Gilbert, R. O., S. T. Shin, C. L. Guard, and H. N. Erb. 1998. Incidence of endometritis and effects on reproductive performance of dairy cows. Theriogenology 49:251.

Gilbert, R. O., S. T. Shin, C. L. Guard, H. N. Erb, and M. Frajblat. 2005. Prevalence of endometritis and its effects on reproductive performance of dairy cows. Theriogenology 64:1879-1888.

Kasimanickam, R., T. F. Duffield, R. A. Foster, C. J. Gartley, K. E. Leslie, J. S. Walton, and W. H. Johnson. 2004. Endometrial cytology and ultrasonography for the detection of subclinical endometritis in postpartum dairy cows. Theriogenology 62:9-23.

Kasimanickam, R., T. F. Duffield, R. A. Foster, C. J. Gartley, K. E. Leslie, J. S. Walton, and W. H. Johnson. 2005. A comparison of the cytobrush and uterine lavage techniques to evaluate endometrial cytology in clinically normal postpartum dairy cows. Can. Vet. J. 46:255-259.

LeBlanc, S. 2005. Overall reproductive performance of Canadian dairy cows: Challenges we are facing. Adv. Dairy Technol. 17:137-157.

LeBlanc, S. 2007. Economics of improving reproductive performance in dairy herds. Adv. Dairy Technol. 19:201-214.

LeBlanc, S. J., T. F. Duffield, K. E. Leslie, K. G. Bateman, G. P. Keefe, J. S. Walton, and W. H. Johnson. 2002. Defining and diagnosing postpartum clinical endometritis and its impact on reproductive performance in dairy cows. J. Dairy Sci. 85:2223-2236.

Maldonado, G., and S. Greenland. 1993. Simulation study of confounder-selection strategies. Am. J. Epidemiol. 138:923-936.

McDougall, S., R. Macaulay, and C. Compton. 2007. Association between endometritis diagnosis using a novel intravaginal device and reproductive performance in dairy cattle. Anim. Reprod. Sci. 99:9-23.

Runciman, D. J., G. A. Anderson, and J. Malmo. 2009. Comparison of two methods of detecting purulent vaginal discharge in postpartum dairy cows and effect of intrauterine cephapirin on reproductive performance. Aust. Vet. J. 87:369-378.

Santos, N. R., H. B. Roman, and R. O. Gilbert. 2006. The use of leukocytes esterase reagent strips for diagnosis of subclinical endometritis in dairy cows. Theriogenology 66:666-667.

Williams, E. J., D. P. Fischer, D. U. Pfeiffer, G. C. W. England, D. E. Noakes, H. Dobson, and I. M. Sheldon. 2005. Clinical evaluation of postpartum vaginal mucus reflects uterine bacterial infection and the immune response in cattle. Theriogenology 63:102-117. 